\title{
Una pedagogía crítica en la universidad: pedagogía de acción y de transformación social ${ }^{1}$
}

\author{
Annie Couëdel
}

Nicole Blondeau ${ }^{2}$

\section{RESUMEN}

El dispositivo de la pedagogía de proyectos se caracteriza por su anclaje en la realidad concreta de los estudiantes y su entorno social. Los proyectos que éstos emprenden permiten la no ruptura entre el interior y el exterior así como salir de la artificialidad inherente a las situaciones pedagógicas: el lenguaje es utilizado como una herramienta de intervención en los dispositivos sociales que ellos planean transformar. La participación en la vida universitaria y más allá de ella conduce a los estudiantes a cuestionar lo que está en juego, a asumir una posición, a situarse mejor en el ajedrez de la política y a desarrollar un espíritu crítico, condición sine qua non del ejercicio de la democracia.

\section{Palabras clave}

Dispositivo de pedagogía de proyectos, confrontación interior/exterior, actores sociales, la lengua como herramienta de intervención social, arquitectura pedagógica, cooperación, democracia, educación dialógica, intervención, inserción.

\section{ABstract}

The device of pedagogy of projects is characterized by its anchoring in the concrete reality of the students and their social environment. The projects that they initiate allow the non-rupture between the inside and the outside and to leave the artificiality inherent in teaching situations: language is used here as a tool of intervention in the social devices they plan to transform. Participation in university life and beyond leads the students to question what's at stake, to form an opinion, to gain a better position in the political chessboard and to develop a critical spirit, a sine qua non of the experience of democracy.

\section{KEYWORDS}

Device of pedagogy of projects, outer/inner confrontation, social actors, language as tool of social intervention, pedagogical architecture, cooperation, democracy, dialogical education, intervention, insertion.

Traducido por Gabriela Gooley.

2 Conferencistas en Ciencias de la Educación, Departamento de Comunicación, Francés como Lengua Extranjera, Formación Permanente, Universidad de París 8. 


\section{Introducción}

\author{
El proyecto fundador de Vincennes \\ era politico y las formas que tomó la enseñanza \\ derivaban, en su mayoria, \\ de las opciones politicas iniciales. \\ Reflexionar hoy sobre las formas actuales \\ de la práctica pedagógica lleva necesariamente \\ a un retorno a lo politico. \\ G. Berger y M. Courtois
}

(La lettre de París 8, abril-mayo 1992, Pédagogie et politique)
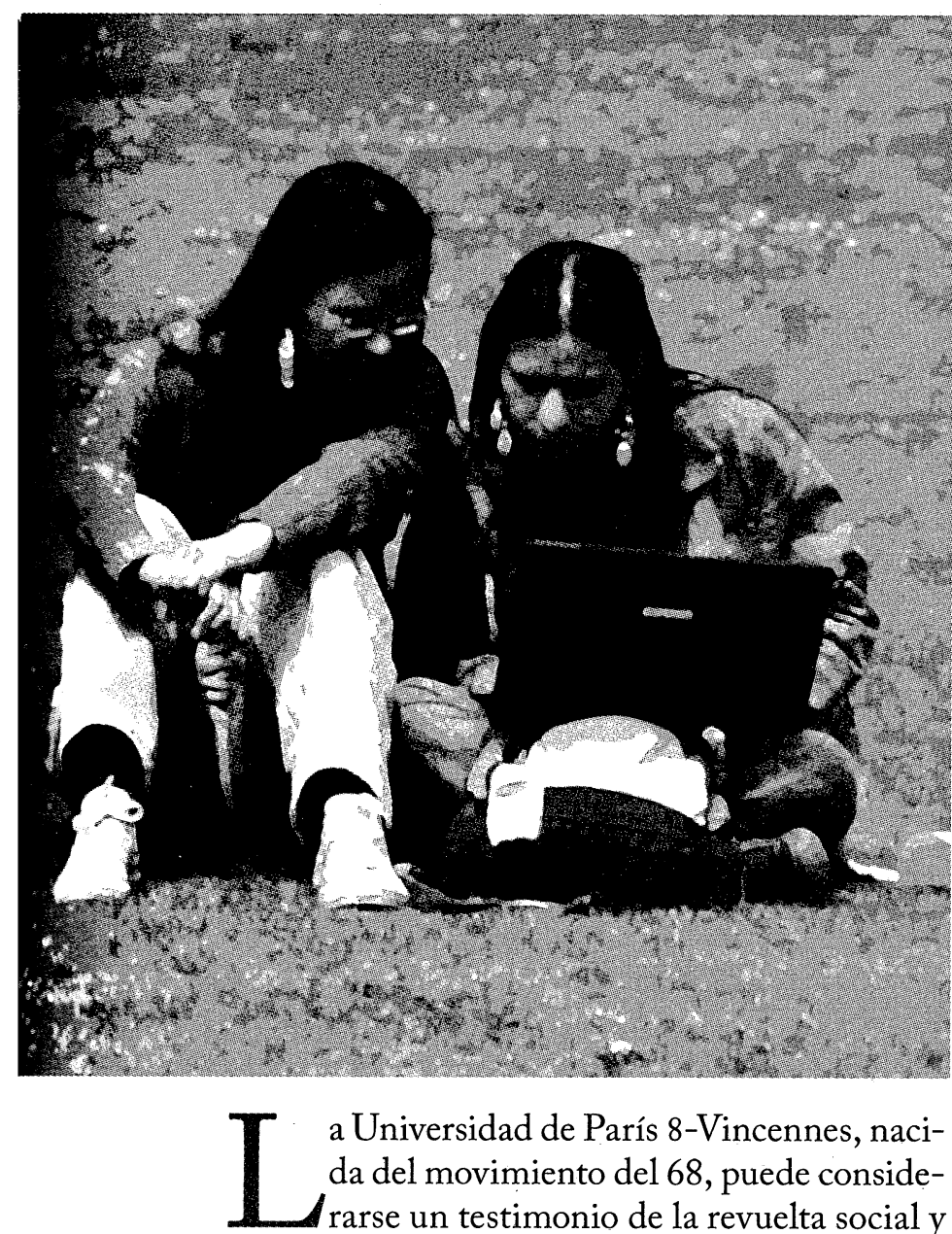

a Universidad de París 8-Vincennes, nacida del movimiento del 68, puede considerarse un testimonio de la revuelta social y una respuesta del Gobierno a la crisis de la institución universitaria (Couëdel, 1999). Vincennes se convierte en un terreno de debate y de discusión permanente. Los estudiantes y una parte importante de los profesores, que habían estado junto a ellos para oponerse a la enseñanza tradicional, rechazan categóricamente los cursos ex cátedra y la relación pedagógica autoritaria para librarse de la sujeción impuesta por una forma de transmisión del conocimiento. Imaginan un nuevo tipo de funcionamiento en el que el profesor ya no es la única referencia y quieren hacerse cargo de su propio aprendizaje. De lo que se trata entonces es que estudiantes y profesores reflexionen conjuntamente sobre nuevos dispositivos pedagógicos. Se hizo costumbre discutir, en cada curso, el tema político del momento, llegando a rechazar las barreras que separan las preocupaciones universitarias, el papel del profesor y el de individuo social.

En este contexto A. Couëdel (1981) puso en práctica, en los años 70 en la Universidad de Vincennes, en él marco del Departamento de Francés como Lengua Extranjera, el dispositivo de pedagogía de proyectos presentado aquí. El objetivo era que, de entrada, los estudiantes extranjeros tuvieran la palabra y jugaran un papel de autores-actores en el juego institucional. La idea central fue definir, al lado del objetivo que desde el punto de vista institucional es el único que cuenta, aprender la lengua, otro objetivo aparentemente alejado: la realización de un proyecto común, elegido y definido por los estudiantes, relacionado con la realidad social circundante. El postulado era que lejos de significar una pérdida de tiempo, este procedimiento permitía más eficazmente lograr el primer objetivo. Esto explica que algunos años más tarde se aceptara en los cursos a estudiantes franceses. Las situaciones que el dispositivo pedagógico pone en marcha, por diversas e imprevisibles que puedan ser, tienen todas en común la puesta a prueba de un tipo de relación particular en la que por una parte el lenguaje y, en forma más general, la comunicación son utilizados como saberes y saber-hacer ajustados a los requerimientos de un contexto preciso. E1 lenguaje no está concebido como un sistema de signos "neutros" que los individuos producen, sino como una manera de inscribirse socialmente, de pensar el mundo y las relaciones sociales (Bautier, 1995). La lengua se aprende también para intervenir en dispositivos sociales que se pretende modificar o, por lo menos, que se pretende manejar. Como dice Bourdieu, la competencia implica también el poder de imponer la recepción de lo que uno dice puesto 
que no se busca simplemente ser comprendido, sino también que a uno le crean, le obedezcan, lo respeten, lo distingan. De ahí la definición completa de competencia como derecho à la palabra (Bourdieu, 1982). De esta manera la lengua es considerada en su ejercicio cívico como herramienta de intervención social.

\section{El dispositivo}

El dispositivo reposa en una arquitectura específica que combina el aporte de los subgrupos (grupos-proyecto), que trabajan con autonomía fuera del campo de la sala de clase, y del grupo grande, un vaivén entre subgrupos y grupo grande, entre exterior e interior. El objetivo del grupo grande en sesiones plenarias es de servir como marco para las síntesis que surgen de los grupos-proyecto, para la evaluación, la crítica, la ejercitación para hablar en público, la autocorrección, la práctica del debate, incluso sobre temas de actualidad. La ritualización de la arquitectura pedagógica sirve de referencia espaciotemporal y juega un papel esencial en la estructuración de un conjunto complejo. Esta forma de proceder es el marco en el interior del cual pueden ejercerse las reglas de "moral social" mínimas: la escucha, el respeto por el otro, la cooperación, la puntualidad, la asiduidad, la responsabilidad individual y colectiva. Todos estos principios están sometidos a la prueba del sentido. Las reglas mencionadas no funcionan como fórmulas mágicas, sino como valores democráticos que garantizan un saber ser con otros, para que el proyecto tenga éxito. En el centro de este dispositivo se encuentra la persona, su inscripción social y su posibilidad de intervención.

Los proyectos son colectivos y constituyen la manifestación de iniciativas inéditas vinculadas a la realidad social. Tienen como objetivo intervenir en los dispositivos sociales que se pretende transformar. El cambio sólo puede tener lugar si hay confrontación, incluso enfrentamiento, entre concepciones divergentes. Por lo tanto, la crítica y la toma de conciencia social están en el centro del proyecto, porque a los valores dominantes de determinados dispositivos sociales se oponen otros valores. El objetivo es que progresivamente los estudiantes participen de la vida universitaria, que cuestionen lo que está en juego por medio de la práctica, que tomen partido, que hablen sobre los problemas contemporáneos, a fin de posicionarse mejor en el tablero político, de tener un papel en la universidad y en la ciudad, incluso más allá de las fronteras (Couëdel, 1997).

\section{El lenguaje no está concebido como un sistema de signos "neutros" que los individuos producen, sino como una manera de inscribirse socialmente, de pensar el mundo y las relaciones sociales.}

Son muchos los proyectos que vieron la luz del día. A continuación mencionaremos algunos: constitución de una red internacional interuniversitaria de encuentros y de investigación sobre las nociones de interculturalidad e interdisciplinariedad, "Le triangle de l'Ecumeur"; creación de asociaciones ley 1901 como el Centre Interculturel de Vincennes à Saint-Denis (CIvD ${ }^{31}$, Centro Intercultural de Vincennes en Saint-Denis), TVNerf (una televisión en París 8) o Erê brasil (defensa de los educadores y de los niños de la calle de Río de Janeiro); edición de una revista semestral, Echo-graphie, y de otras publicaciones como Beurk, La Gouve; participación en un seminario de preparación del contrato cuadrienal que determina la política de la universidad; creación de un sitio en Internet, Étudiants sans-papiers

\footnotetext{
El civd es una asociación creada en 1901 y dirigida por los estudiantes. Fue creado en 1984, luego de un primer festival intercultural organizado en el marco de los talleres de elaboración de proyectos del Departamento de Comunicación/Francés Lengua Extranjera de París 8. Nació a partir del deseo de recordarle a París 8 su vocación de internacionalismo, dirigido sobre todo al Tercer Mundo, para que nuestra universidad no sea sólo un lugar funcional de estudios, sino una realidad social donde se establecen vínculos activos y vivos entre personas diferentes. Permite que los proyectos que surgen de los talleres tengan una continuidad. Es un lugar con vocaciones múltiples: inserción y creación, formación, producción y edición, investigación intercultural y expansión al extranjero.

El sitio es http://www.ipt.univ-paris8.fr/ civd/parcours.
} 
(Estudiantes sin papeles), http://mageos.ifrance. com/sans-papiers, en los años 2000-2001, en plena lucha por la regularización de los estudiantes extranjeros; organización de un festival intercultural anual, de jornadas sobre temas específicos en la universidad (Info sida, en relación con la medicina preventiva, "Lib'air ta pensée", referidas al intercambio comercial justo); instalación de una biblioteca en una ciudad de Togo, "A la rencontre d'un village... Cultures croisées a Akoumapé”... Tantos proyectos, fruto de la iniciativa de los estudiantes, originados en una misma dinámica, un mismo impulso: "hacer algo útil", "tener la posibilidad de actuar", expresiones que encontramos frecuentemente en los bitácora de los estudiantes (Blondeau y Couëdel, 2000).

El anclaje del dispositivo en la realidad social lleva a los participantes a observar en perspectiva, a descubrir cómo funciona la institución, qué es lo que está en juego y quiénes son los actores. El proyecto está concebido como un modelo organizador que va a estructurar las relaciones con el exterior. Para que tome forma, es necesario que haya debates entre los participantes a fin de llegar a un "entendimiento $\mathrm{mu}$ tuo" (según la concepción de Habermas), dado que el grupo-proyecto es particularmente heterogéneo (estudiantes de culturas, origen social, edad, ideología y nivel universitario diferentes), pero también enfrentamiento y negociaciones con las autoridades, descubrimiento y apreciación de las relaciones de fuerza entre grupos más o menos informales, cuyas posturas influencian la toma de decisiones. Los promotores de proyectos aprenderán a identificar todos los códigos, normas y relaciones de poder que emanan de la institución, utilizando al mismo tiempo estrategias de intervención que obligarán al sistema, en el mejor de los casos, a cooperar o, por lo menos, a reaccionar.

En esta pedagogía lo que justifica la acción del educador no es un contenido preconstruido ni conocimientos clasificados y jerarquizados que hay que transmitir. Aquí podría decirse que su papel está "desplazado". Algunos estudiantes constatan, a veces con sorpresa y otras con rabia, la actitud de "retiro" del educador. Pueden sentir como indiferencia la actitud del profesor de no tomar parte cuando hay un mal funcionamiento en el grupo o cuando aparecen problemas interpersonales. Esta actitud tiene su justificación en la voluntad de no caer en la trampa de la relación dual educador/alumno, con los riesgos de identificación, delegación, corrección y confusión que ella implica, y de respetar la mediación a través del proyecto y el ritual establecido en los talleres. Este papel "descentrado" no significa ausencia de participación. El educador es el garante del buen funcionamiento del dispositivo, es el que acompaña los proyectos, es la persona-recursos en lo que se refiere al acceso a las redes "de afiliación" y a las redes institucionales. Es el que pone en relación a los portadores de proyectos con las instancias o personas "facilitadoras", da las pistas para los pedidos de subvenciones, sugiere posibles alternativas. Teje un vínculo entre lo que parece funcionar de manera autónoma. En determinados momentos puede convertirse en participante del proyecto, igual que los estudiantes, y luego dejar de participar cuando el proyecto está en vías de lograrse. En este caso existe una especie de alianza estratégica entre los participantes, que juntan sus herramientas de análisis para comprender las trabas impuestas por el entorno, tratar de servirse de ellas, de pasarlas por alto, de suprimirlas. Comienza entonces un verdadero trabajo de objetivación del funcionamiento institucional y social, que deja en claro los acuerdos tácitos entre actores cuyas posiciones parecían contrapuestas, desenmascara falsas alianzas o descubre alianzas reales.

Ya lo hemos dicho, la pedagogía de proyectos, tal como la concebimos aquí, se inscribe en una historia y una lucha política que suponen un compromiso y elecciones en lo que se refiere a la concepción de la enseñanza. En los talleres se hacen explícitos el anclaje histórico y los fundamentos filosóficos y pedagógicos del dispositivo. Esta aclaración rompe con la ilusión de que habría conocimientos válidos intrínsecamente, es decir, separados del contexto social en el que se construyeron y transmitieron. El hecho de decir que no hay ni enseñanza ni educador neutros da a los estudiantes la posibilidad de tomar posición y no es raro que las posiciones tomadas sean objeto de críticas, a veces virulentas. A fines del semestre 2000-2001 algunos estudiantes les reprocharon a los profesores el no haber intervenido cuando 
los proyectos iban hacia un callejón sin salida y el no haber sido lo suficientemente precisos en cuanto a los objetivos de la pedagogía, mientras que otros consideraron que el "abandono" en el que los habían dejado les había permitido forjar sus propias armas y que el fracaso también era formador. Aquí, lo interesante no es subrayar la acusación a una práctica sino el hecho de que los intercambios se hayan desviado rápidamente de su "objetivo" y empezaran a circular en el grupo. El enfrentamiento dejó de ser alumnos/educadores para pasar a ser alumnos/alumnos, inmersos en un espacio de libertad de palabra, usando las reglas del debate democrático.

La actitud crítica respecto del entorno nace y se desarrolla a partir de estas formas de enfrentamiento interno y externo, dando lugar al distanciamiento y cuestionamiento de.las normas sociales interiorizadas. Retomamos aquí el concepto de "concientización" de Paulo Freire, para quien la educación no puede pensarse independientemente del poder que la constituye, ni separada de la realidad concreta en la cual se inserta. Para Paulo Freire, todo aprendizaje debe estar estrechamente ligado a la situación concreta vivida por el alumno. El punto de partida consiste en admitir que la libertad y la crítica son dones esenciales de la vida humana. El grupo de trabajo e intercambios o "círculo de cultura" tiene como objetivo principal el estudio del lenguaje en el contexto de una práctica social libre y crítica. Es a través de la educación como acto político y la "concientización" que el hombre podrá transformar la realidad, su realidad. Freire opone su punto de vista a la que él denomina educación bancaria, es decir, la que se limita a depositar un conocimiento ya construido. Para Freire, toda la educación debe ser dialógica, basada en los conocimientos y la experiencia de los alumnos y fuertemente arraigada en los contextos culturales. Una pedagogía de la libertad implica la adopción de actitudes democráticas, en el marco de acciones compartidas por todos: educadores y alumnos (Freire, 1977).

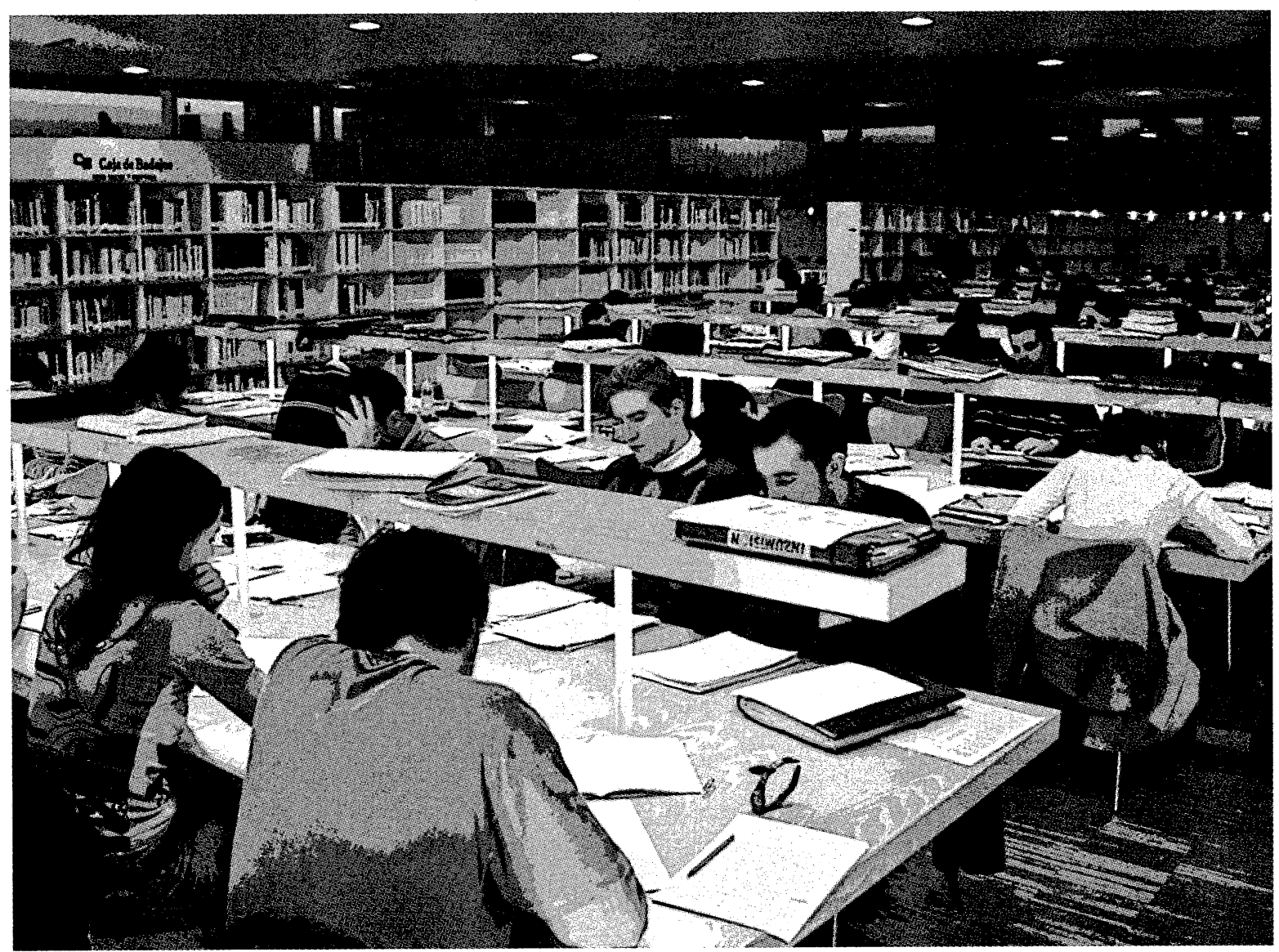


Consideramos pertinente hacer referencia aquí a un artículo de Inés Barbosa de Olivera (1995) basado en las investigaciones que dieron origen a su tesis Pour une pédàgogie de la transformation sociale. Sus trabajos se apoyan en Habermas y su concepción de la moral es entendida como un conjunto de principios interactivos definidos por los miembros del grupo social (y no como un conjunto de reglas trascendentales), asociada a los conceptos de igualdad y libertad, lo cual permite hablar sobre la cuestión del pluralismo de manera concreta. Esta concepción de la moral implica una aceptación a priori de todas las diferencias de concepción moral y social. Siempre según Inés Barbosa de Olivera, que pone en primer lugar la cooperación y la búsqueda de un acuerdo mutuo y no el ejercicio del poder de los que saben sobre los que no saben:

"La gestión democrática, con todas sus dificultades y el hecho de compartir responsabilidades que supone, debe ser el objetivo de toda comunidad escolar. El acuerdo mutuo en la escuela significa la puesta en práctica de un proyecto colectivo de reconstrucción estructural y pedagógica. A nivel de las clases de la escuela, el establecimiento de un funcionamiento democrático en el cual los estudiantes participan activamente en la elección de los contenidos y las estrategias pedagógicas, a través de debates en los que las opiniones y conocimientos diversos son respetados y puestos a prueba, deja de ser, en este marco, una concesión hecha por educadores "simpáticos" para convertirse en un imperativo que se impone a todo educador comprometido en un proyecto de democratización de la sociedad" (340).

Nuestro proyecto pedagógico se entiende como el ejercicio de un vínculo social que se define por la negativa: no autoritario, no piramidal y no institucional, donde el lenguaje no es solamente un "objeto de intelección" sino también un "instrumento de poder y de acción" (Bourdieu, 1982). Nuestra concepción de la educación es un camino de emancipación de los sujetos sociales "que transforman el mundo al mismo tiempo que se transforman".

\section{Los proyectos}

En noviembre de 1995, los estudiantes que el año anterior habían participado en los talleres "Concepción y realización de proyectos" quisieron aportar su contribución al seminario de preparación del contrato cuadrienal titulado "Quelle université voulons-nous?" (¿Qué universidad queremos nosotros?), en el grupo de reflexión "La universidad, lugar de vida y cultura". Entonces se sumaron a los profesores y personal iATos en las reuniones semanales de preparación, que tuvieron lugar durante más de seis meses. Siendo participantes activos del Centro Intercultural Vincennes en Saint-Denis (CIVD), habían encontrado muchos problemas y trabas administrativas. Conjuntamente con las asociaciones culturales existentes, investigaron las relaciones que éstas tenían con la universidad y demostraron la necesidad de una verdadera vida asociativa autónoma en París 8. El balance de los años de funcionamiento del CIVD (1985 a 1995), redactado por su tesorero, Christophe Forgeot y adjuntado al informe de la investigación, dice lo siguiente:

De 1985 a 1992 y sin tener en cuenta los años de "sueño forzado" de la asociación por decisión del presidente de la universidad (que terminaron en la prohibición brutal y autoritaria del festival intercultural anual), puedo decir sin reparos y con la adecuada perspectiva que el civd cumplió con éxito el papel que sus fundadores le habían asignado, es decir: promover la vida en la universidad París 8. Gracias a esta vía interna, la universidad París 8 no ha sido solamente un lugar en el que los estudiantes vienen a "consumir" conocimientos y a estudiar, sino también un lugar en el que todos, cualquiera fuera su posición en la universidad, pueden encontrarse, descubrirse, conocerse, enriquecerse en el contacto con el Otro. La universidad tomaba otra dimensión, en la cual las nociones de creación, de intercambio, de interculturalidad, de tolerancia, tenían importancia [...] además de ser un lugar de enseñanza se convertía en un lugar de vida intercultural y creativa". 
El texto continúa haciendo referencia a las numerosas acciones que se llevaron a cabo durante el periodo y denuncia el hecho de que el cIvD haya perdido parte de su "libertad de acción" en la universidad. Esto tuvo como consecuencia "la modificación de su modo de funcionamiento en detrimento de la vivacidad y la rapidez de acción”. Termina de este modo:

Me incorporé a esta asociación porque les permitía a los estudiantes crear en conjunto, cualquiera fuera el color de su piel, su religión o sus opiniones políticas. $Y$ deseo fervientemente que el civD, verdadero símbolo de interculturalidad en un momento en el que hay tantas tensiones nacionalistas, símbolo de fraternidad en la creación y el emprendimiento en un momento de apatía ambiente, digo entonces que [deseo que] el civd retome los medios de acción que han contribuido a mejorar la calidad de vida de los usuarios de París 8 durante todos estos años. El civd fue fundado con el fin de cuidar el mejor aspecto de Vincennes. Actuemos para que pueda proseguir libremente su función sin obstáculos, hoy y en el futuro (1995).

La participación en el seminario de preparación del contrato cuadrienal 1996-2000 tendrá repercusiones inmediatas. Los estudiantes descubrirán, gracias a uno de sus representantes elegidos, la existencia "confidencial" del FAve, fondo "exclusivamente destinado al mejoramiento de la vida estudiantil". La existencia de este fondo, reunido con descuentos hechos en el pago de la matrícula de inscripción, fue ocultada. Representa un presupuesto considerable, utilizado por ejemplo en la refacción de instalaciones cuyo financiamiento es responsabilidad del Estado. La lucha será larga para obligar a la universidad a que cree una línea presupuestaria específica, pero la motivación y la combatividad de los estudiantes son más fuertes dado que han logrado organizar desde 1984 un festival intercultural anual que la universidad jamás apoyó financieramente. Al mismo tiempo, un grupo-proyecto de la UE prepara un encuentro intercultural, "Le triangle de 1'Ecumeur" (Couëdel, Blondeau y Kalentieva, 2001), conjuntamente con estudiantes y profesores del Instituto Universitario de Lenguas de
Irkoutsk (Rusia) y de la Universidad de Torreón (México), que tendrá lugar en la Universidad de West Georgia (Estados Unidos). Para este encuentro el cIvD busca subsidios, que no obtendrá, pero una línea presupuestaria propia se creará al comienzo de las clases el año siguiente.

Los estudiantes subrayan el hecho de que no se tomen en cuenta sus iniciativas, pero sobre todo insisten en que ha habido violencia institucional hacia ellos, por ejemplo, la prohibición del festival intercultural anual y luego la destrucción de los archivos del CIvD ${ }^{42}$. Esta violencia revela el miedo de la institución de que se perturbe un funcionamiento que es, a fin de cuentas, satisfactorio para los profesores y la administración. Por el momento, ya no está en la agenda una universidad como lugar para hablar, discutir y reflexionar sobre el mundo contemporáneo. No obstante, ante los fracasos masivos en el primer ciclo, ante la ausencia de un proyecto que integre las distintas federaciones ¿no deberíamos, en conjunto, tratar de crear una dinámica de coconstrucción de sentido, de hacer una articulación entre el "adentro" y el "afuera"?

Pierre Bourdieu decía en los años 70 que la situación escolar considerada como situación lingüística de un tipo particular ejerce una formidable censura sobre todos los que anticipan las posibilidades de ganancia y de pérdida que tienen dada la capacidad de la que disponen. $Y$ el silencio de algunos no es más que el interés (1978), constatación que sigue siendo actual. Un aspecto a menudo descuidado es la falta de motivación de los estudiantes, resultado de la ausencia de anclaje de los estudios en la vida y del sentido que la universidad les da a sus vidas. Bernard Charlot, en un trabajo presentado en ese mismo seminario ${ }^{5}$, decía:

un número creciente de estudiantes vive la universidad como un lugar para adquirir diplomas y no como un lugar de cultura, la vive como un lugar funcional específico en una vida

\footnotetext{
4 Informe interno del seminario de la universidad. (17-18 de noviembre de 1995): Quelle université voulons-nous?, París 8.

5 Ver nota anterior.
} 
dispersa entre varios espacios más que como el territorio de base de lo cotidiano. Por lo tanto es más difícil para la universidad cumplir una función cultural más allá de los cùrsos y ser un lugar de vida, es decir un lugar de elaboración de sentido (del mundo, de la vida, de la sociedad, de la relación con los otros, de la relación consigo mismo) [...] La universidad podría volver a ser un lugar de vida y de cultura si contribuyera a producir sentido -más allá de los cursos, donde se supone que lo hace (1995: 187).

Para hacerlo es necesario establecer vínculos nuevos entre la universidad y su entorno, un vínculo entre la universidad y las instituciones en las que hoy se tratan cuestiones sociales y filosóficas fundamentales. Siempre según B. Charlot, "los estudiantes no tienen las referencias culturales que teníamos nosotros, no obstante tienen una experiencia del mundo, de la vida y de la actividad social que a menudo es más rica y diversa que la nuestra" (1995: 189). ¿Cómo tener en cuenta esta experiencia del mundo, articular esos saberes a otros más académicos para lograr coherencia y producción de sentido? En otras palabras, ¿qué proyecto político hay que darle a la universidad?

Durante el año universitario 2000-2001 nuestra universidad conoció un periodo de tensiones graves. Hubo una ocupación por parte de estudiantes sin documentos, que duró seis meses y que perturbó enormemente la vida universitaria. La mayor parte de la comunidad universitaria no sintió la necesidad de buscar las causas de la rebelión y dejó que la situación siguiera su curso. Para salir del callejón sin salida se hizo intervenir a la policía y se encontraron chivos expiatorios para calmar la situación. Esto dio como resultado que se acusara a cuatro jóvenes tunecinos que serán juzgados, corren el riesgo de ser sentenciados a penas de prisión y de ser expulsados a su país de origen, sobre el cual no podemos afirmar que sea del todo democrático.

A veces es en los momentos de "crisis", de grandes perturbaciones, que los estudiantes descodifican con más agudeza el funcionamiento institucional. En ese contexto de lucha, una estudiante chilena de los talleres-proyecto creó un sitio en Internet denominado Étudiants sanspapiers (estudiantes sin papeles), compuesto de once partes: historia de nuestra universidad, condiciones de admisión en las universidades, aportes de profesores, de administrativos, de sindicatos, comunicados de prensa, revisión de la prensa, testimonios, peticiones y llamados para reaccionar. La estudiante explica así su objetivo: "Este sitio pretende ser un espacio de información que permita un diálogo constructivo para que la universidad mantenga siempre el espíritu utópico de la Vincennes de los años 70". En la página de presentación se pone de relieve lo siguiente: "El saber está hecho para ser compartido sin ninguna distinción", seguido de la historia de las leyes referidas a la admisión de estudiantes extranjeros en Francia y sus repercusiones: disminución de la cantidad de estudiantes (en 1982, 13\%, en 1999, 8\%, incluidos europeos y no europeos), llegada de estudiantes que corren muchos riesgos al venir a Francia sin pasar por el filtro de los servicios culturales franceses del país de origen, etc. En la página dice:

Limitar arbitrariamente el acceso de los estudiantes extranjeros compromete el papel cultural y científico de Francia, su acción económica, el desarrollo de sus universidades y la apertura a la evolución del mundo actual. ¿¿Se puede seguir siendo el país de los derechos humanos cerrándose a los otros?

La presentación termina con esta declaración: 
Este sitio ha sido creado por personas preocupadas por las medidas discriminatorias contra las poblaciones inmigrantes que tomaron los diferentes gobiernos franceses (Francia, patria de los derechos humanos, ¿dónde vas?). Su existencia depende de todas y todos nosotros, de nuestras ganas de disponer de información lo más objetiva posible, antes del establecimiento de un diálogo sereno y quizás, del surgimiento de soluciones [...] estas -son nuestras "pretensiones" [...]

¿Hubiera sido posible evitar esta crisis? Henry Lefèbvre pensaba que si no se ejercía la función crítica se iba hacia la crisis. La presencia de un número relativamente elevado de estudiantes provenientes de los países del sur provoca en algunos actores de nuestra universidad una actitud de rechazo. Sin duda, al introducir heterogeneidad y complejidad, estos estudiantes "molestan", crean malestar. Esta noción de "molestia" tal como la entiende Patrice Ville (2001) nunca fue cuestionada y la universidad enfrenta a menudo situaciones "críticas". La respuesta por medio de la fuerza, en vez de un debate democrático, deja siempre para más tarde el examen de los problemas de fondo que tiene la sociedad: las relaciones norte/sur, ciudad/alrededores, culturas dominantes/culturas dominadas. Tantas cuestiones que sin duda encontrarían respuestas más satisfactorias, por lo menos en París 8 , si los estudiantes franceses/extranjeros en sus cursos pudieran participar en proyectos comunes. Esto podría contribuir, quizás, a afrontar algunos desafíos de la sociedad. Como dice Jacques Ardoino (2000), se trata de desarrollar una inteligencia de las contradicciones y del conflicto, para la cual no preparan necesariamente las formaciones tradicionales, se trata de restaurar la comunicación entre los actores, es decir "el reconocimiento de la heterogeneidad, de la legitimidad de las diferencias entre los participantes, que deriva de sus respectivos backgrounds (historia, formación, experiencias) e implicancias, como también de los intereses en función de los cuales se ubican los unos con respecto a los otros".

Otros dos proyectos relativos a las relaciones norte/sur se llevaron a cabo durante el año universitario 2000-2001. En el primero, “Lib'air ta pensée", se llevaron a cabo tres jornadas, en la universidad, sobre el "comercio justo". "Es esencial una toma de conciencia para entender el sentido de nuestras iniciativas", escriben los estudiantes en la carpeta de presentación. "El comercio debe adquirir conciencia [...] Nos plantea interrogantes acerca de las relaciones norte/sur, la producción de masas, como también sobre los factores sociales y ecológicos, que deberían ser el punto principal de todas las actividades sociales: la producción del hombre para el hombre". Uno de los resultados de estas tres jornadas fue acercarse al crous (Centro Regional de Obras Universitarias y Sociales) para que proponga a los estudiantes de la universidad productos de "comercio justo".

La experiencia fue muy enriquecedora $y$ este curso me ha permitido hacer cosas de las que no me creía capaz, además de descubrir el universo de la universidad desde otra perspectiva. ¡Fascinante! [...] Este espíritu de crítica y también de permanente cuestionamiento de la institución en la que nos encontramos me parece interesante y me permite por lo tanto prestar más atención y estar más cerca de la universidad, también me hace sentir más estudiante, me hace sentir más dentro del sistema y me da una maravillosa impresión de poder.

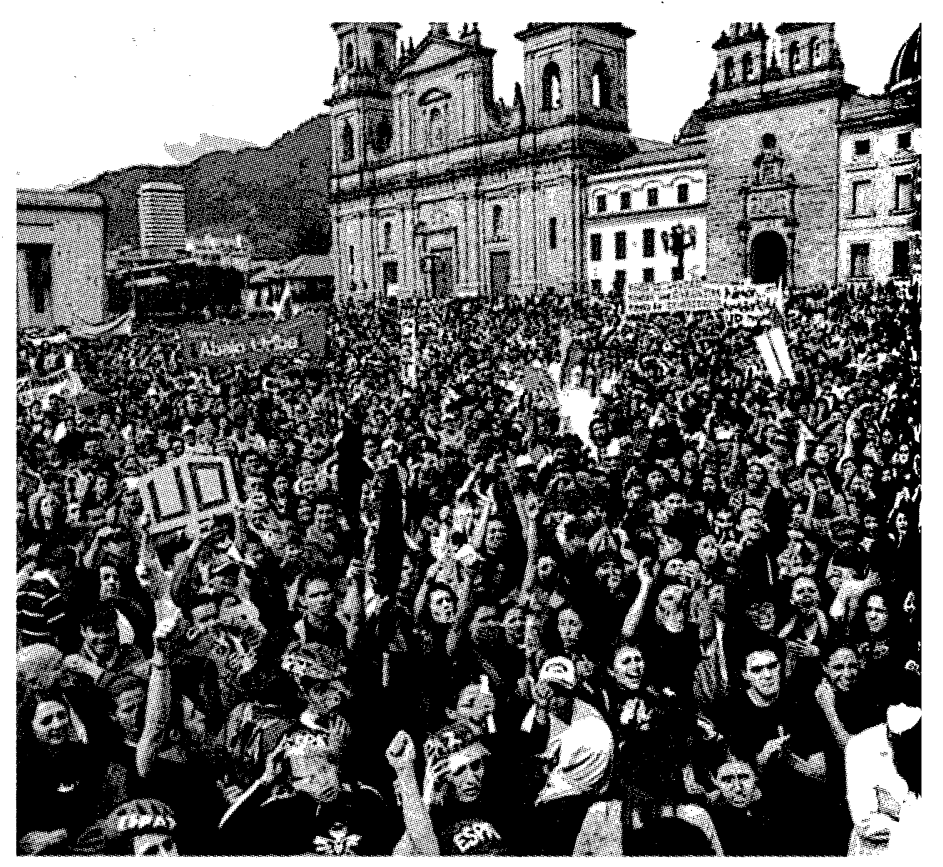


concluye Juliette, una de las actrices del proyecto.

En el segundo, "A la rencontre d'un village... Cultures croisées a Akoumapè" (Al encuentro de un pueblo... Culturas cruzadas en Akoumapé), se trata de la creación de una biblioteca en un "pueblo" de treinta mil habitantes en Togo. Los estudiantes elaboraron el proyecto durante un año: constitución de dossiers para pedir subvenciones, contactos con los habitantes del pueblo, obtención de autorizaciones por parte de los jefes tradicionales, colecta de libros. Ocho estudiantes, entre ellos uno togolés, iniciador del proyecto, fueron en agosto a trabajar en el pueblo. Para hacer llegar los 1.500 libros obtenidos -principalmente para niños y adolescentes- contaron con el apoyo de la agencia intergubernamental para la francofonía. El local destinado a la biblioteca fue restaurado y puesto en funcionamiento en tres semanas, los libros fueron clasificados y se organizó un sistema de préstamo. La inauguración se llevó a cabo con gran despliegue, contó con la presencia de los habitantes, de los jefes tradicionales y de una delegación prefectoral. En el marco de las festividades, se representó una obra de teatro que los estudiantes escribieron y pusieron en escena conjuntamente con los jóvenes del pueblo. Luego los jefes tradicionales donaron un terreno para la construcción de un centro social destinado a los jóvenes. Nuevamente los estudiantes participaron en esta iniciativa.

¿Cuál fue la contribución de FAVE para estos dos proyectos? Mientras que "Lib'air ta pensée" recibió una subvención, al proyecto Akoumapé le rechazaron dos veces un pedido de ayuda, con el pretexto siguiente: "la comisión FAVE colectiva se interesa prioritariamente en las acciones que tienen un impacto sobre el sitio de París 8" (12/03/2001). Fue necesario un amplio movimiento de apoyo para que los estudiantes obtuvieran finalmente una subvención, pero no para el proyecto, sino para "la realización de una exposición" sobre Togo al regresar de allí. Esto significa una interpretación muy estrecha de la circular ministerial que subraya Lucette Colin, profesora de Ciencias de la Educación y directora de la UFR 8, en la carta que dirigió a los miembros de la comisión:
Me parece [que este proyecto] presenta un interés particular en relación con una de las características de nuestra universidad: la tasa de estudiantes extranjeros [y africanos] más elevada de Francia. Llevar a cabo, aunque no sea más que a título experimental, un proyecto de vinculación entre los estudiantes de París 8 y la realidad de un pueblo africano, proponer una actividad de tipo ONG, con la organización de un retorno a nivel de los estudiantes de Saint-Denis, es una idea muy interesante. Sin duda la comisión que otorga los créditos de FAVE puede considerar que un proyecto de esa índole no contribuye al mejoramiento de la vida estudiantil y que financiar ese proyecto es una decisión de tipo político. Se puede pensar también que esta iniciativa contribuye a considerar diferentemente la acogida de nuestros estudiantes extranjeros en el sentido de una cooperación, lo que tendría repercusiones directas en la relación que los estudiantes tienen con la vida universitaria en París 8.

Nuestra concepción de la educación, ya se habrá entendido, tiene "una dimensión definitivamente política en la medida que el proyecto es llevado a cabo por actores, autores, por personas que no están retenidas en un programa con un jefe de programa que impone modelos" (Berger, 1998: 34). Se apoya en una demanda de emancipación de sujetos sociales, capaces de adaptarse a las nuevas realidades sociales y económicas o de combatirlas. El dispositivo de pedagogía de proyectos estimula el surgimiento de acciones de transformación y permite acoger lo real, pensarlo, analizarlo, someterlo colectivamente a la prueba del sentido. Se trata de provocar en los estudiantes un cuestionamiento permanente acerca del mundo, un preguntarse sobre el lugar que ocupan y su compromiso, sin ocultar lo que parece antagonista ni neutralizar lo que se presenta como concurrente. Es el acceso a la complejidad lo que llama, según Edgar Morin, a una reforma del pensamiento, es decir una reforma que establezca el principio de relación, acercando lo que hasta el momento se concebía de forma separada y a veces repelente (Morin, 1998). 
Para no concluir, dejaremos la palabra a uno de los actores de los encuentros de "Le triangle de l'Ecumeur":

Esta pedagogía permite, justamente, que las personas se encuentren y tomen conciencia de sus diferencias, pero además jexiste esa red formidable de relaciones que puede tejerse! $¡$ En ese sentido nos parece algo absolutamente esencial! Por mi parte pienso que sin duda jes una respuesta formidable a la exclusión, probablemente también a la indiferencia! [...] No es muy interesante para nosotros funcionar aislados jes necesario abrirse! Por lo tanto, esta idea de red un poco "satelital" nos permite, justamente, hacerlo y difundir nuestra existencia. Lo interesante para nosotros es que, cuantos más seamos en ir en esta dirección, mayor será la comprensión mutua. Yo ubico esto verdaderamente en un plano también humano, porque nos encontramos en un fin de siglo algo difícil $\mathrm{y}$ a veces es esencial volver a centrar las cosas y poner en contexto las desviaciones, podría decirse, culturales, las exclusiones culturales, etc. Aquí me ubico en una perspectiva casi francófona, pero también es cierto que en Estados Unidos se puede observar que hay una especie de ruptura, de muro entre las diferentes comunidades. Nada pasa a través, hay muchas comunidades, pero no hay comunicación entre ellas. Si esto pudiera ayudar, justamente, a derribar esos muros (Khelouz, 1997). Ó

\section{Bibliografía}

Ardoino, J. (2000), Les avatars de l'éducation, Paris, PUF.

Bautier, E. (1995), Pratiques langagières, pratiques sociales. De la sociolinguistique à la sociologie du langage, París, L'Harmattan.

Barbosa de Oliveira, I. (1993), Pour une Université de transformation sociale [tesis de grado], Université des Sciences Humaines de Strasbourg I.

- (1995), "Place et rôle de la démocratisation à l'école selon Habermas", en Les Sciences de l'Education, núm. 2, vol. 21, pp. 223-436.
Blondeau, N. y Couëdel A. (2000), "Pédagogie de projet, journal de bord et appréhension de la diversité culturelle", en Dialogues et cultures, núm. 44, pp. 36-51.

Bourdieu, P. (1978, marzo), "Ce que parler veut dire”, en Le français aujourd'bui, núm. 41, pp. 4-20.

- (1982), Ce que parler veut dire, París, Fayard.

Charlot, B. (1977), Du rapport au savoir. Eléments pour une théorie, París, Anthropos.

Couëdel, A. (1981), "Vivre la langue, de la communication à la langue", en Champs éducati$f s$, núm. 3, revista publicada por el Groupe de Recherche sur l'Education, Centre de recherche, Université Paris 8, pp.37-76.

- (1995), "Cultures. Pedagogie. Politique" [anexo a las actas del seminario del 17 y 18 de noviembre de 1995], en Quelle université voulons-nous, París, Paris 8.

- (1997), "Par delà les frontières", en Europe plurilingue, núm. 12/13 pp. 38-46.

- (1999), [conferencia dada en Bogotá el 18 de noviembre de 1999] "Movimiento estudiantil de 68 en Francia y el desarrollo histórico de la universidad de Paris 8" en el marco del simposio continental: Movimientos universitarios en América Latina, siglo XX. (Consultar en el sitio Étudiants sans papiers: (http://mageos.ifrance.com/sans-papiers).

-; Blondeau, N. y Kalentieva, T. (2001), "Interculturalité et lien social. Un dispositif de rencontres internationales: Le triangle de l'Ecumeur', en Dialogues et cultures, núm. 45, pp. 388-393.

Freire, P. (1977), Pédagogie des opprimés, París, FM, Petite Collection, Maspéro.

Habermas, J. (1987), La théorie de l'agir communicationnel, Tomos I y II, París, Fayard.

Khelouz, N., 3er encuentro del Triangle de l'Ecumeur, julio-agosto del 97 en la universidad París 8.

Morin, E. y Bonnefoy, Y. (1998), Articuler les savoirs. L'enseignement de la poésie, París, Ministère de l'Education Nationale.

Nuestro agradecimiento a nuestros colegas y amigos Guy Berger y Maurice Courtois por la atención y el apoyo que nos brindaron. 